\title{
СХОДИМОСТЬ ИНТЕРПОЛЯЦИОННЫХ СПЛАЙНОВ ЧЕТВЕРТОЙ СТЕПЕНИ ${ }^{1}$
}

\begin{abstract}
Ю. С. Волков
Рассмотрена задача интерполяции сплайнами четвертой степени по схеме Марсдена. Показано, что при вычислении интерполяционного сплайна через коэффициенты разложения его второй производной по $L_{1}$-нормализованным В-сплайнам второй степени приходим к системе линейных уравнений относительно выбранных параметров с пятидиагональной матрицей, имеющей диагональное преобладание по столбцам. Наличие диагонального преобладания позволяет эффективно с практической точки зрения вычислить определяемые параметры и установить сходимость процесса интерполяции сплайнов по Марсдену для любой функции класса $C^{1}$ на произвольной последовательности сеток без каких-либо ограничений. В схеме Марсдена считается, что задана сетка узлов сплайна, а точки интерполяции выбираются строго посередине. Установленные результаты переносятся на случай интерполяции сплайнами четвертой степени по схеме Субботина (сетки данных и узлов сплайна меняются местами). Здесь система уравнений относительно коэффициентов разложения третьей производной по $L_{\infty}$-нормализованным В-сплайнам будет иметь диагональное преобладание и сходимость процесса интерполяции будет иметь место для любой интерполируемой функции класса $C^{3}$.
\end{abstract}

Ключевые слова: сплайны четвертой степени, интерполяция, сходимость, матрицы с диагональным преобладанием.

Yu. S. Volkov. Convergence of quartic interpolation splines.

The problem of interpolation by quartic splines according to Marsden's scheme is considered. It is shown that the calculation of an interpolating spline in terms of the coefficients of expansion of its second derivative in $L_{1}$-normalized quadratic B-splines yields a system of linear equations for the chosen parameters. The matrix of the system is pentadiagonal and has a column diagonal dominance, which makes it possible to efficiently calculate the required parameters and establish the convergence of the spline interpolation process according to Marsden's scheme for any function from the class $C^{1}$ on an arbitrary sequence of grids without any constraints. In Marsden's scheme, it is assumed that a knot grid is given and the interpolation nodes are chosen strictly in the middle. The established results are transferred to the case of interpolation by quartic splines according to Subbotin's scheme (the node grid and knot grid are swapped). Here the system of equations for the coefficients of expansion of the third derivative in $L_{\infty}$-normalized B-splines has a diagonal dominance, and the interpolation process converges for any interpolated function from the class $C^{3}$.

Keywords: quartic splines, interpolation, convergence, diagonally dominant matrices.

MSC: 41A05, 41A15, 41A25

DOI: $10.21538 / 0134-4889-2019-25-2-67-74$

\section{Введение}

В настоящее время основным решением задачи интерполяции являются сплайны. Классические интерполяционные сплайны нечетной степени минимизируют функционал "энергии”, и сетка узлов совпадает с сеткой данных. Однако при перенесении такой конструкции на сплайны четной степени можно столкнуться с проблемами существования [1], поэтому здесь принято сетки узлов сплайна и данных выбирать перемежающимися. Распространены две схемы интерполяции: узлы сплайна выбираются строго посередине между точками интерполяции (схема Субботина) или, наоборот, точки интерполяции выбираются строго посередине между узлами сплайна (схема Марсдена).

\footnotetext{
${ }^{1}$ Работа выполнена в рамках государственного задания ИМ СО РАН (проект № 0314-2016-0013) и при частичной финансовой поддержке РФФИ и ННИО (проект № 19-51-12008).
} 
Задача интерполяции сплайнами невысоких степеней (параболическими и кубическими) хорошо изучена, установлены оценки погрешности интерполяции, разработаны эффективные алгоритмы построения сплайнов (см. [2-5]). Для таких сплайнов системы линейных уравнений относительно каких-либо параметров являются трехдиагональными, и зачастую матрицы имеют диагональное преобладание, что обеспечивает эффективность вычисления этих параметров и изучения всех свойств. Однако системы уравнений для сплайнов более высоких степеней усложняются, диагональное преобладание в известных на данный момент соответствующих системах уравнений отсутствует, что не позволяет гарантировать устойчивое вычисление определяемых параметров и затрудняет получение оценок погрешности и изучение сходимости процессов интерполяции. Для сплайнов пятой степени сходимость вторых и третьих производных установлена из свойств вполне неотрицательных матриц [6; 7].

В настоящей работе для интерполяционных сплайнов четвертой степени по схеме Марсдена явно выписаны системы линейных уравнений относительно коэффициентов разложения второй производной определяемого сплайна по В-сплайнам второй степени, матрицы полученных систем уравнений имеют диагональное преобладание по столбцу. Наличие столбцевого диагонального преобладания позволяет установить сходимость процесса интерполяции для функций класса $C^{1}$ и предложить надежный и устойчивый метод построения интерполяционного сплайна четвертой степени по Марсдену.

\section{1. Система линейных уравнений}

Пусть на отрезке $[a, b]$ задана сетка точек, являющихся узлами сплайнов,

$$
\Delta: a=x_{0}<x_{1}<\ldots<x_{n}=b,
$$

причем на краях добавим по два совпадающих узла

$$
x_{-2}=x_{-1}=x_{0}, \quad x_{n}=x_{n+1}=x_{n+2} .
$$

Середины промежутков сетки $\Delta$ образуют еще одну сетку

$$
\delta: a=\xi_{0}<\xi_{1}<\ldots<\xi_{n}<\xi_{n+1}=b, \quad \xi_{i}=\left(x_{i-1}+x_{i}\right) / 2, \quad i=-1, \ldots, n+2,
$$

задающую набор точек, в которых известны значения некоторой гладкой функции $f \in C^{1}[a, b]$, т. е. $f_{i}=f\left(\xi_{i}\right), i=0, \ldots, n+1$. Кроме того, в крайних точках отрезка $[a, b]$ известны значения производных $f_{a}^{\prime}=f^{\prime}(a), f_{b}^{\prime}=f^{\prime}(b)$.

Рассматриваем задачу интерполяции известных значений сплайном четвертой степени $s \mathrm{c}$ узлами на сетке $\Delta$ минимального дефекта (гладкости $C^{3}$ ) по схеме Марсдена. Таким образом, должны выполняться условия интерполяции

$$
s\left(\xi_{i}\right)=f_{i}, \quad i=0, \ldots, n+1,
$$

и краевые условия

$$
s^{\prime}(a)=f_{a}^{\prime}, \quad s^{\prime}(b)=f_{b}^{\prime} .
$$

Сплайн с такими краевыми условиями принято называть полным.

Для вычисления интерполяционного сплайна необходимо найти какие-либо параметры. В работе [8] предложено в качестве определяемых параметров выбирать коэффициенты разложения одной из производных искомого сплайна по В-сплайнам подходящей степени. Сейчас такими параметрами считаем коэффициенты разложения второй производной нашего сплайна четвертой степени, являющейся в свою очередь сплайном второй степени на сетке $\Delta$, по $L_{1}$ нормализованным В-сплайнам второй степени $M_{i}=M_{i, 3, \Delta}$, т. е. определяемыми параметрами являются $\beta_{-2}, \ldots, \beta_{n-1}$ и

$$
s^{\prime \prime}(x)=\sum_{i=-2}^{n-1} \beta_{i} M_{i}(x) .
$$


На каждом промежутке $\left[x_{i}, x_{i+1}\right], i=0, \ldots, n-1$, сетки $\Delta$ отличны от нуля только 3 базисных сплайна второй степени. Выпишем для $x \in\left[x_{i}, x_{i+1}\right]$ их представление

$$
\begin{aligned}
M_{i-2}(x) & =\frac{3\left(x_{i+1}-x\right)^{2}}{\left(x_{i+1}-x_{i-2}\right)\left(x_{i+1}-x_{i-1}\right)\left(x_{i+1}-x_{i}\right)}, \\
M_{i-1}(x) & =\frac{3\left(x-x_{i-1}\right)\left(x_{i+1}-x\right)}{\left(x_{i+2}-x_{i-1}\right)\left(x_{i+1}-x_{i-1}\right)\left(x_{i+1}-x_{i}\right)}+\frac{3\left(x_{i+2}-x\right)\left(x-x_{i}\right)}{\left(x_{i+2}-x_{i-1}\right)\left(x_{i+2}-x_{i}\right)\left(x_{i+1}-x_{i}\right)}, \\
M_{i}(x) & =\frac{3\left(x-x_{i}\right)^{2}}{\left(x_{i+3}-x_{i}\right)\left(x_{i+2}-x_{i}\right)\left(x_{i+1}-x_{i}\right)} .
\end{aligned}
$$

В соответствии с работой [8] система линейных уравнений относительно вектора неизвестных $\boldsymbol{\beta}=\left(\beta_{-2}, \ldots, \beta_{n-1}\right)^{T}$ имеет вид

$$
\boldsymbol{A} \boldsymbol{\beta}=\boldsymbol{d},
$$

где $\boldsymbol{A}=\left(a_{i, j}\right)$ - пятидиагональная матрица размера $(n+2) \times(n+2)$ с элементами, определяемыми формулами

$$
a_{i, j}=\int_{x_{j-3}}^{x_{j}} M_{j-3}(\tau) N_{i-2}(\tau) d \tau, \quad i, j=1, \ldots, n+2,
$$

вектор правой части $\boldsymbol{d}=\left(d_{1}, \ldots, d_{n+2}\right)^{T}$ имеет компоненты

$$
\begin{aligned}
d_{1} & =f\left[\xi_{0}, \xi_{1}\right]-f_{a}^{\prime}, \\
d_{i} & =f\left[\xi_{i-1}, \xi_{i}\right]-f\left[\xi_{i-2}, \xi_{i-1}\right], \quad i=2, \ldots, n+1, \\
d_{n+2} & =f_{b}^{\prime}-f\left[\xi_{n}, \xi_{n+1}\right],
\end{aligned}
$$

функции $N_{i}=N_{i, 2, \delta}$ являются $L_{\infty}$-нормализованными В-сплайнами первой степени с узлами на сетке $\delta$. Сплайн $N_{i}$ имеет носитель $\left[\xi_{i}, \xi_{i+2}\right]$, и на каждом интервале сетки $\delta$ отличны от нуля только два таких сплайна. Выпишем для $x \in\left[\xi_{i}, \xi_{i+1}\right]$ их представление

$$
N_{i-1}(x)=\frac{\xi_{i+1}-x}{\xi_{i+1}-\xi_{i}}, \quad N_{i}(x)=\frac{x-\xi_{i}}{\xi_{i+1}-\xi_{i}} .
$$

\section{2. Разрешимость задачи интерполяции}

Введем обозначения

$$
\begin{gathered}
h_{i}=x_{i+1}-x_{i}, \quad i=-2, \ldots, n+1 ; \\
\mu_{i}=\frac{h_{i-1}}{h_{i-1}+h_{i}}, \quad \lambda_{i}=1-\mu_{i}, \quad i=0, \ldots, n ; \\
\theta_{i}=\frac{h_{i-1}}{x_{i+2}-x_{i-1}}, \quad \eta_{i}=\frac{h_{i}}{x_{i+2}-x_{i-1}}, \quad \zeta_{i}=1-\theta_{i}-\eta_{i}, \quad i=-1, \ldots, n .
\end{gathered}
$$

Теперь формулы представления базисных функций можно записать более компактно. При $x=x_{i}+t h_{i}, 0 \leqslant t \leqslant 1, B$-сплайны первого семейства имеют вид

$$
\begin{aligned}
M_{i-2}(x) & =\frac{3(1-t)^{2} \lambda_{i}}{x_{i+1}-x_{i-2}}, \\
M_{i-1}(x) & =\frac{3\left[\left(\mu_{i}+t \lambda_{i}\right)(1-t)+\left(\lambda_{i+1}+(1-t) \mu_{i+1}\right) t\right]}{x_{i+2}-x_{i-1}}, \\
M_{i}(x) & =\frac{3 t^{2} \mu_{i+1}}{x_{i+3}-x_{i}} .
\end{aligned}
$$


Для базисных функций второго семейства при $x=x_{i}+t h_{i}$ имеем

$$
\begin{aligned}
N_{i-1}(x) & =(1-2 t) \lambda_{i}, & & t \in[0,1 / 2], \\
N_{i}(x) & =\mu_{i}+2 t \lambda_{i}, & & t \in[0,1 / 2], \\
N_{i}(x) & =2(1-t) \mu_{i+1}+\lambda_{i+1}, & & t \in[1 / 2,1], \\
N_{i+1}(x) & =(2 t-1) \mu_{i+1}, & & t \in[1 / 2,1] .
\end{aligned}
$$

В результате интегрирования для ненулевых элементов матрицы $\boldsymbol{A}$ системы (1) получаем значения

$$
\begin{aligned}
a_{1,1}= & 17 / 32, \quad a_{1,2}=\mu_{1}\left(6+\lambda_{1}\right) / 32, \quad a_{1,3}=\mu_{1} \theta_{1} / 32 \\
a_{2,1}= & \left(14+\lambda_{1}\right) / 32, \quad a_{2,2}=\left(17+3 \mu_{1}+3 \mu_{1} \lambda_{1}\right) / 32, \\
a_{2,3}= & {\left[14 \theta_{1}+\left(3+3 \lambda_{1}+\lambda_{1} \lambda_{2}\right) \eta_{1}\right] / 32, \quad a_{2,4}=\lambda_{1} \mu_{2} \theta_{2} / 32 } \\
a_{i, i-2}= & \lambda_{i-3} \mu_{i-2} \zeta_{i-4} / 32, \\
a_{i, i-1}= & {\left[\left(3+\mu_{i-3} \mu_{i-2}+3 \mu_{i-2}\right) \eta_{i-3}+\left(14+\lambda_{i-2} \lambda_{i-1}\right) \zeta_{i-3}\right] / 32, } \\
a_{i, i}= & {\left[17+\eta_{i-2}\left(3+3 \mu_{i-2}+2 \mu_{i-2} \lambda_{i-1}+3 \lambda_{i-1}\right)\right] / 32, } \\
a_{i, i+1}= & {\left[\left(14+\mu_{i-2} \mu_{i-1}\right) \theta_{i-1}+\left(3+3 \lambda_{i-1}+\lambda_{i-1} \lambda_{i}\right) \eta_{i-1}\right] / 32, } \\
b_{i, i+2}= & \lambda_{i-1} \mu_{i} \theta_{i} / 32, \\
& i=3, \ldots, n ; \\
a_{n+1, n-1}= & \lambda_{n-2} \mu_{n-1} \zeta_{n-3} / 32, \\
a_{n+1, n}= & {\left[\left(3+\mu_{n-2} \mu_{n-1}+3 \mu_{n-1}\right) \eta_{n-2}+\left(14+\lambda_{n-1} \lambda_{n}\right) \zeta_{n-2}\right] / 32, } \\
a_{n+1, n+1}= & \left(17+3 \mu_{n-1} \lambda_{n-1}+3 \lambda_{n-1}\right) / 32, \quad a_{n+1, n+2}=\left(\mu_{n-1}+14\right) / 32 ; \\
a_{n+2, n}= & \lambda_{n-1} \zeta_{n-2} / 32, \quad a_{n+2, n+1}=\lambda_{n-1}\left(6+\mu_{n-1}\right) / 32, \quad a_{n+2, n+2}=17 / 32 .
\end{aligned}
$$

Теорема 1. Полный сплайн четвертой степени по Марсдену с узлами на сетке $\Delta$, интерполирующий произвольные заданные значения на сетке $\delta$, всегда существует и единствен.

Д о к а з а т е л ь с т в о. Нами вычислены явные выражения для всех компонент системы уравнений (1). Покажем, что матрица этой системы имеет строгое диагональное преобладание по столбцам. Найдем количественные величины $r_{i}$ диагонального преобладания для каждого столбца матрицы $\boldsymbol{A}$. Для первых двух столбцов имеем

$$
\begin{aligned}
r_{1} & =a_{1,1}-a_{2,1}-a_{3,1}=17 / 32-\left(14+\lambda_{1}\right) / 32-\mu_{1} / 32=1 / 16, \\
r_{2} & =a_{2,2}-a_{3,2}-a_{4,2}-a_{1,2} \\
& =\left(17+3 \mu_{1}+3 \mu_{1} \lambda_{1}\right) / 32-\left(3+3 \mu_{1}^{2}+11 \lambda_{1}+\lambda_{1}^{2} \lambda_{2}\right) / 32-\lambda_{1}^{2} \mu_{2} / 32-\mu_{1}\left(6+\lambda_{1}\right) / 32 \\
& =\left(1+3 \mu_{1}+3 \mu_{1} \lambda_{1}\right) / 16>1 / 16 .
\end{aligned}
$$

Теперь вычислим $r_{i}$ для внутренних столбцов $(i=3, \ldots, n)$ :

$$
r_{i}=a_{i, i}-a_{i-2, i}-a_{i-1, i}-a_{i+1, i}-a_{i+2, i}=
$$




$$
\begin{aligned}
= & {\left[17+\eta_{i-2}\left(3+3 \mu_{i-2}+2 \mu_{i-2} \lambda_{i-1}+3 \lambda_{i-1}\right)\right] / 32 } \\
& -\lambda_{i-3} \mu_{i-2} \theta_{i-2} / 32-\left[\left(14+\mu_{i-3} \mu_{i-2}\right) \theta_{i-2}+\left(3+3 \lambda_{i-2}+\lambda_{i-2} \lambda_{i-1}\right) \eta_{i-2}\right] / 32 \\
& -\left[\left(3+\mu_{i-2} \mu_{i-1}+3 \mu_{i-1}\right) \eta_{i-2}+\left(14+\lambda_{i-1} \lambda_{i}\right) \zeta_{i-2}\right] / 32-\lambda_{i-1} \mu_{i} \zeta_{i-2} / 32 \\
= & {\left[3-\mu_{i-2} \theta_{i-2}-\lambda_{i-1} \zeta_{i-2}+\eta_{i-2}\left(11+3 \mu_{i-2}+2 \mu_{i-2} \lambda_{i-1}\right.\right.} \\
& \left.\left.+3 \lambda_{i-1}-3 \lambda_{i-2}-\lambda_{i-2} \lambda_{i-1}-\mu_{i-2} \mu_{i-1}-3 \mu_{i-1}\right)\right] / 32 \\
= & {\left[2+\lambda_{i-2} \theta_{i-2}+\mu_{i-1} \zeta_{i-2}+\eta_{i-2}\left(6+5 \mu_{i-2}+4 \mu_{i-2} \lambda_{i-1}+5 \lambda_{i-1}\right)\right] / 32 } \\
= & {\left[1+\eta_{i-2}\left(3+3 \mu_{i-2}+2 \mu_{i-2} \lambda_{i-1}+3 \lambda_{i-1}\right)\right] / 16>1 / 16 . }
\end{aligned}
$$

И, наконец, для последних двух столбцов матрицы $\boldsymbol{A}$ получаем

$$
\begin{aligned}
r_{n+1}= & a_{n+1, n+1}-a_{n-1, n+1}-a_{n, n+1}-a_{n+2, n+1} \\
= & \left(17+3 \mu_{n-1} \lambda_{n-1}+3 \lambda_{n-1}\right) / 32-\lambda_{n-2} \mu_{n-1}^{2} / 32 \\
& -\left(3+\mu_{n-2} \mu_{n-1}^{2}+11 \mu_{n-1}+3 \lambda_{n-1}^{2}\right) / 32-\lambda_{n-1}\left(6+\mu_{n-1}\right) / 32 \\
= & \left(1+3 \mu_{n-1} \lambda_{n-1}+3 \lambda_{n-1}\right) / 16>1 / 16, \\
r_{n+2}= & a_{n+2, n+2}-a_{n, n+2}-a_{n+1, n+2}=17 / 32-\lambda_{n-1} / 32-\left(\mu_{n-1}+14\right) / 32=1 / 16 .
\end{aligned}
$$

Вычисления показывают, что величина диагонального преобладания в первой и последней строках матрицы равна 1/16, а в остальных больше, поэтому система всегда невырождена и имеет единственное решение. Теорема доказана.

\section{3. Сходимость процесса интерполяции}

Рассмотрим на отрезке $[a, b]$ последовательность сеток $\{\Delta\}$, удовлетворяющую условию $h=\max _{i} h_{i} \rightarrow 0$ и которой подчинена последовательность сеток $\{\delta\}$.

Теорема 2. Для любой функиии $f \in C^{1}[a, b]$ и произвольной последовательности сеток, удовлетворяющей условию $h \rightarrow 0$, если полные сплайны четвертой степени s интерполируют $f$ в точках сеток $\delta$ (по схеме Марсдена), то последовательность s' сходится равномерно $\kappa f^{\prime}$.

Д о к а з а т е л ь с т в о. Для интерполяционных сплайнов четной степени по Марсдену в работе [8, теорема 6] установлена оценка

$$
\left\|s^{\prime}-f^{\prime}\right\|_{C} \leqslant K\left\|A^{-1}\right\| \omega\left(f^{\prime} ; h\right)
$$

с некоторой константой $K$. Здесь $\|\boldsymbol{B}\|$ есть тах-норма матрицы $\boldsymbol{B}=\left(b_{i, j}\right), \omega(g ; h)$ - модуль непрерывности функции $g$.

Ясно, что проблема сходимости процесса интерполяции в этом случае сводится к оценке нормы обратной матрицы системы уравнений (1). Для матриц с диагональным преобладанием существует [9] довольно простой способ оценки нормы обратной матрицы

$$
\left\|\boldsymbol{B}^{-1}\right\| \leqslant \max _{i} \frac{1}{R_{i}}
$$

где

$$
R_{i}=b_{i, i}-\sum_{j \neq i}\left|b_{i, j}\right|
$$


А для элементов обратной матрицы к $\boldsymbol{B}$ имеется [5, теорема Д.6] оценка

$$
\left|\hat{b}_{i, j}\right| \leqslant \frac{1}{b_{j, j}} \cdot \max _{k} \frac{b_{k, k}}{R_{k}}\left(1-\min _{k} \frac{R_{k}}{b_{k, k}}\right)^{|i-j| / m},
$$

где $m=\max \left\{|i-j|: b_{i, j} \neq 0\right\}-$ ширина ленты ленточной матрицы.

Однако матрица нашей системы уравнений имеет лишь диагональное преобладание по столбцам, поэтому оценка (3) будет применима лишь к транспонированной матрице $\boldsymbol{A}^{T}$. Но при доказательстве теоремы 1 мы установили оценки величин диагонального преобладания в каждом столбце матрицы $\boldsymbol{A}$, поэтому если обозначить

$$
\sigma=1-\min _{k} \frac{r_{k}}{a_{k, k}}, \quad D=\max _{k} \frac{1}{a_{k, k}} \cdot \max _{k} \frac{a_{k, k}}{r_{k}},
$$

то для элементов обратной матрицы $\boldsymbol{A}^{-1}=\left(\hat{a}_{i, j}\right)$ системы уравнений (1) неравенство (4) перепишется в виде

$$
\left|\hat{a}_{i, j}\right| \leqslant \frac{1}{a_{i, i}} \cdot \max _{k} \frac{a_{k, k}}{r_{k}} \sigma^{|i-j| / m} \leqslant D \sigma^{|i-j| / m} .
$$

Тогда для нормы обратной матрицы $\boldsymbol{A}$ справедлива оценка

$$
\left\|\boldsymbol{A}^{-1}\right\|=\max _{i} \sum_{j}\left|\hat{a}_{i, j}\right| \leqslant D\left(1+2 \sigma^{1 / m}+2 \sigma^{2 / m}+\ldots\right) \leqslant D \frac{1+\sigma^{1 / m}}{1-\sigma^{1 / m}} .
$$

В нашем случае $m=2, D=16, \sigma=15 / 17$, поэтому в итоге получаем

$$
\left\|\boldsymbol{A}^{-1}\right\| \leqslant 512
$$

т. е. тах-норма обратной матрицы системы уравнений (1) для любой сетки $\Delta$ ограничена абсолютной константой, следовательно, правая часть в неравенстве (2) стремится к нулю при $h \rightarrow 0$. Таким образом, теорема 2 доказана.

Следствие. Если полный сплайн четвертой степени s с узлами на произвольной сетке $\Delta$ интерполирует по схеме Марсдена функцию $f \in C^{1}[a, b]$, то имеют место неравенства

$$
\left\|s^{(k)}-f^{(k)}\right\|_{C} \leqslant K_{k} h^{1-k} \omega\left(f^{\prime} ; h\right), \quad k=0,1,
$$

с абсолютными константами $K_{0}$ и $K_{1}$.

Для интерполяционных сплайнов нечетной степени известно (см. обзор Тр. Ин-та математики и механики УрО РАН, 2014, Т. 20, № 1. С. 52-67), что безусловная сходимость имеет место только для двух средних производных. По-видимому, для сплайнов четной степени ситуация аналогичная, и мы предполагаем, что для сплайнов четвертой степени по схеме Марсдена будет иметь место сходимость процесса интерполяции еще для вторых производных, если интерполируемая функция принадлежит классу $C^{2}$.

\section{4. О схеме интерполяции по Субботину}

Впервые задача интерполяции сплайнами четной степени была рассмотрена Ю.Н.Субботиным [10] и М.Марсденом [11] для сплайнов второй степени. Хотя рассмотренные конструкции суть две существенно различные схемы интерполяции, автор [8] установил связь этих схем. Матрицы определяющих систем уравнений относительно коэффициентов разложения по Всплайнам $k$-й производной интерполяционного сплайна степени $2 m$ по схеме Марсдена являются транспонированными к матрицам систем уравнений относительно также коэффициентов разложения по В-сплайнам, но уже $(2 m+1-k)$-й производной интерполяционного сплайна той же степени $2 m$ по схеме Субботина. 
Таким образом, рассмотрение задачи интерполяции сплайном четвертой степени по схеме Субботина приводит к тому, что если выбрать определяемыми параметрами коэффициенты разложения третьей производной искомого сплайна по $L_{\infty}$-нормализованным В-сплайнам, то матрица получаемой системы линейных уравнений будет являться транспонированной к рассмотренной в этой работе и, следовательно, будет иметь диагональное преобладание по строкам.

В [8] также установлена связь условий сходимости процессов интерполяции по схемам Субботина и Марсдена. Если на какой-либо последовательности сеток $\{\Delta\}$ имеет место равномерная сходимость $s^{(k)}$ к $f^{(k)}$ для $f \in C^{k}$ по схеме Марсдена для сплайнов степени $2 m$, то на этой же последовательности сеток будет иметь место сходимость $s^{(2 m-k)}$ к $f^{(2 m-k)}$ для $f \in C^{2 m-k}$ по схеме Субботина. Верно и наоборот. Тогда результат теоремы 2, перенесенный на сплайны по Субботину будет таким.

Теорема 3. Для любой функиии $f \in C^{3}[a, b]$ и произвольной последовательности сеток, удовлетворяющей условию $h \rightarrow 0$, если полные сплайны четвертой степени $s$ с узлами на сетках $\delta$ интерполируют $f$ в точках сеток $\Delta$ (по схеме Субботина), то последовательность $s^{\prime \prime \prime}$ сходится равномерно $\kappa f^{\prime \prime \prime}$.

Для интерполяционных сплайнов четвертой степени по схеме Субботина мы также предполагаем, что без каких-либо ограничений на сетки безусловная сходимость процесса интерполяции будет иметь место для вторых производных, если интерполируемая функция принадлежит классу $C^{2}$.

Заметим, что сходимость без ограничений на сетки при интерполяции функции класса $C$ сплайном четвертой степени по Субботину невозможна [12].

\section{СПИСОК ЛИТЕРАТУРЫ}

1. Ahlberg J. H., Nilson E. N., Walsh J. L. Best approximation and convergence properties of higherorder spline approximations // J. Math. Mech. 1965. Vol. 14, no. 2. P. 231-243.

2. Ahlberg J. H., Nilson E. N., Walsh J. L. The theory of splines and their applications. N Y: Acad. Press, 1967. $284 \mathrm{p}$.

3. Стечкин С. Б., Субботин Ю. Н. Сплайны в вычислительной математике. М.: Наука, 1976. 248 с.

4. Boor C. de. A practical guide to splines. N Y: Springer, 1978. 392 p.

5. Завьялов Ю.С., Квасов Б.И., Мирошниченко В.Л. Методы сплайн-функций. М.: Наука, 1980. 352 c.

6. Boor C. de. On the convergence of odd-degree spline interpolation // J. Approxim. Theory. 1968. Vol. 1, no. 4. P. 452-463. doi: 10.1016/0021-9045(68)90033-6.

7. Волков Ю. С. Вполне неотрицательные матрицы в методах построения интерполяционных сплайнов нечетной степени // Мат. труды. 2004. Т. 7, № 2. С. 3-34.

8. Волков Ю.С. Интерполяция сплайнами четной степени по Субботину и по Марсдену // Укр. мат. журн. 2014. Т. 66, № 7. С. 891-908.

9. Ahlberg J.H., Nilson E. N. Convergence properties of the spline fit // J. Soc. Indust. Appl. Math. 1963. Vol. 11, no. 1. P. 95-104. doi: 10.1137/0111007.

10. Субботин Ю.Н.О кусочно-полиномиальной интерполяции // Мат. заметки. 1967. Т. 1, № 1. C. 63-70.

11. Marsden M. J. Quadratic spline interpolation // Bull. Amer. Math. Soc. 1974. Vol. 80, no. 5. P. 903906.

12. Волков Ю.С. Необходимые условия равномерной сходимости интерполяционных сплайнов четвертой и пятой степеней // Вычисл. системы. / ИМ СО АН СССР. Вып. 93: Методы сплайнфункций. Новосибирск, 1982. С. 30-38. 
Волков Юрий Степанович

д-р физ.-мат. наук, доцент

главный науч. сотрудник

Институт математики им. С. Л. Соболева СО РАН, г. Новосибирск;

профессор

Новосибирский государственный университет, г. Новосибирск

e-mail:volkov@math.nsc.ru

\section{REFERENCES}

1. Ahlberg J.H., Nilson E.N., Walsh J.L. Best approximation and convergence properties of higher-order spline approximations. J. Math. Mech., 1965, vol. 14, no. 2, pp. 231-243.

2. Ahlberg J., Nilson E., Walsh J. The theory of splines and their applications. N Y: Acad. Press, 1967, 284 p. ISBN: 9781483222950 . Translated to Russian under the title Teoriya splainov $i$ ee prilozheniya. Moscow: Mir Publ., 1972, 316 p.

3. Stechkin S.B., Subbotin Yu.N. Splainy $v$ vychislitel'noi matematike [Splines in computational mathematics]. Moscow: Nauka Publ., 1976, 248 p.

4. de Boor C. A practical guide to splines. N Y: Springer, 1978, 392 p. ISBN: 978-3540903567. Translated to Russian under the title Prakticheskoe rukovodstvo po splainam. Moscow: Radio i Svyaz' Publ., 1985, $304 \mathrm{p}$.

5. Zavialov Yu.S., Kvasov B.I., Miroshnichenko V.L. Metody splajn funkcij [Methods of spline-functions]. Moscow: Nauka Publ., 1980, 352 p.

6. de Boor C. On the convergence of odd-degree spline interpolation. J. Approxim. Theory, 1968, vol. 1, no. 4, pp. 452-463. doi: 10.1016/0021-9045(68)90033-6.

7. Volkov Yu.S. Totally positive matrices in the methods of constructing interpolation splines of odd degree. Siberian Adv. Math., 2005, vol. 15, no. 4, pp. 96-125.

8. Volkov Yu.S. Interpolation by splines of even degree according to Subbotin and Marsden. Ukrainian Math. J., 2014, vol. 66, no. 7, pp. 994-1012. doi: 10.1007/s11253-014-0990-z .

9. Ahlberg J.H., Nilson E.N. Convergence properties of the spline fit. J. Soc. Indust. Appl. Math., 1963, vol. 11, no. 1, pp. 95-104. doi: 10.1137/0111007.

10. Subbotin Yu.N. Piecewise-polynomial (spline) interpolation. Math. Notes, 1967, vol. 1, no. 1, pp. 41-45. doi: $10.1007 / \mathrm{BF} 01221723$

11. Marsden M.J. Quadratic spline interpolation. Bull. Amer. Math. Soc., 1974, vol. 80, no. 5, pp. 903-906. ISBN: 10.1090/S0002-9904-1974-13566-4.

12. Volkov Yu.S. Necessary conditions for uniform convergence of interpolationl splines of fourth and fifth degrees. Vychisl. Sist., Novosibirsk, 1982, vol. 93, pp. 30-38 (in Russian).

Received March 1, 2019

Revised March 25, 2019

Accepted April 1, 2019

Funding Agency: This work was supported by the Institute of Mathematics, Siberian Branch of the Russian Academy of Sciences (state contract no. 0314-2016-0013), and partially by the Russian Foundation for Basic Research and the German Research Foundation (project no. 19-51-12008).

Yuriy Stepanovich Volkov, Dr. Phys.-Math. Sci., Prof., Sobolev Institute of Mathematics of the Siberian Branch of the Russian Academy of Sciences, Novosibirsk, 630090 Russia; Novosibirsk State University, Novosibirsk, 630090 Russia, e-mail: volkov@math.nsc.ru .

Cite this article as: Yu. S. Volkov. Convergence of quartic interpolation splines, Trudy Instituta Matematiki i Mekhaniki URO RAN, 2019, vol. 25, no. 2, pp. 67-74. 